\title{
Stationary entanglement of two atoms in a common reservoir
}

\author{
MAN ZhongXiao ${ }^{1 *}$, SU Fang $^{2} \&$ XIA YunJie ${ }^{1}$ \\ ${ }^{1}$ Shandong Provincial Key Laboratory of Laser Polarization and Information Technology, Department of Physics, Qufu Normal University, \\ Qufu 273165, China; \\ ${ }^{2}$ Department of Chemistry and Chemical Engineering, Jining University, Qufu 273155, China
}

Received November 16, 2012; accepted January 19, 2013

\begin{abstract}
We study the dynamics of two entangled atoms interacting with a common structured reservoir. By means of the exact solution of atomic dynamics, we show a novel quantum interference controlled by the relative phase of initial entangled state of the atoms. The quantum interference has a great influence on trapped excited-state population and stationary entanglement of the atoms. In particular, we construct an explicit condition under which atomic stationary entanglement can grow over their initial value.
\end{abstract}

quantum entanglement, quantum interference, common environment

Citation: Man Z X, Su F, Xia Y J. Stationary entanglement of two atoms in a common reservoir. Chin Sci Bull, 2013, 58: 2423-2429, doi: 10.1007/s11434-013-5898-8

Quantum entanglement is one of the most characteristic traits of quantum mechanics and has been recognized as an indispensable resource in realizing the quantum information technologies [1-4]. The fragility of entanglement, however, is one of the reasons that hampers its practical applications. The unavoidable coupling to the surrounding environment(s), whether intentionally or accidentally, is a serious obstacle since it always leads to the loss of open system's information [5]. Worse still, the entanglement may be terminated even in a finite time, a phenomenon called entanglement sudden death [6-9]. Therefore, the study on entanglement dynamics [10-34], namely, to know the residual amount of entanglement after a certain period of time evolution, is relevant to both the fundamental characters and applications of entanglement. In this connection, the issue of entanglement control becomes more and more important and a lot of methods [35-40] have been proposed to prolong the service time of entanglement or to preserve a long-lived stationary entanglement.

The realization of decay mechanism proves to be effective in designing strategy to fight against the deterioration of entanglement. The dissipative interaction between individual quantum system with its environment is a usual mechanism

*Corresponding author (email: manzhongxiao@163.com) spoiling the entanglement of a composite system. An emblematic example of the dissipative interaction is the spontaneous emission of a photon by a two-level atom (qubit) into a vacuum environment of electromagnetic-field modes. Therefore, the methods that can trap the excited-state population would be applicable for entanglement preservation. It has been shown [36] in environments, such as photonic band-gap materials, structured so as to inhibit spontaneous emission of individual atoms, the entanglement of two independent atoms can be trapped. Besides the special structure of the environment, [37] shows the vacuum-induced coherence [41] that can lead to quenching of spontaneous emission in atomic systems can also lead to preservation of atomic entanglement. For several qubits interacting with a same environment [23, 24, 26, 27, 35] and the total Hamiltonian is highly symmetric, there may exist a decoherence-free subspace and the stationary entanglement in the long-time limit can be expected. Focusing on the model that two qubits are coupled to a common structured reservoir, the author in [35] proposed an effective strategy to fight against the deterioration of the entanglement using the quantum Zeno effect. For the same model, Li et al. [26] investigated the exploitation of dipole-dipole interaction between two atoms and atomreservoir coupling strength to suppress disentanglement. It is found for the situation that the dipole-dipole interaction 
exists and the couplings of the atoms to the field are different, the steady state entanglement of the atoms does not exist [26]. Nevertheless, although the dipole-dipole interaction could destroy the asymptotic entanglement, a strong dipole-dipole interaction does suppress disentanglement effects under some conditions [26]. Obviously, in case of the stationary entanglement exists, it is related to the component of decoherence-free state of the initial qubits' state. In other words, different initial states of the qubits will lead to different amount of stationary entanglement. In this work, we shall also explore the model of two atoms interacting with a common reservoir. We pay particular attention to the relative phase of atoms' initial entangled state in influencing the atoms' stationary entanglement. It is found that the suitable choice of relative phase can significantly increase atomic stationary entanglement.

A visualized picture of two atoms interacting with a common reservoir can be depicted by Figure 1. For two entangled atoms with totally one excitation, the single excitation may be distributed among the two atoms with nonzero probabilities. Due to the interactions with the common reservoir, the excitation-emission by one atom will lead to the absorbtion of that excitation by another. As a result, for each atom, two opposite processes of excitation emission and absorbtion will coexist at the same time. A quantum interference effect of these two processes can be expected. Through an exact solution of the model, we show the existence of this quantum interference. We show that the relative phase in atomic initial entangled state will determine the destructive or constructive feature of the interference, which in turn affects the extent of excited-stat population trapped in the atoms as well as their stationary entanglement in the long-time limit. We also give a condition under which atomic stationary entanglement can exceed their initial value.

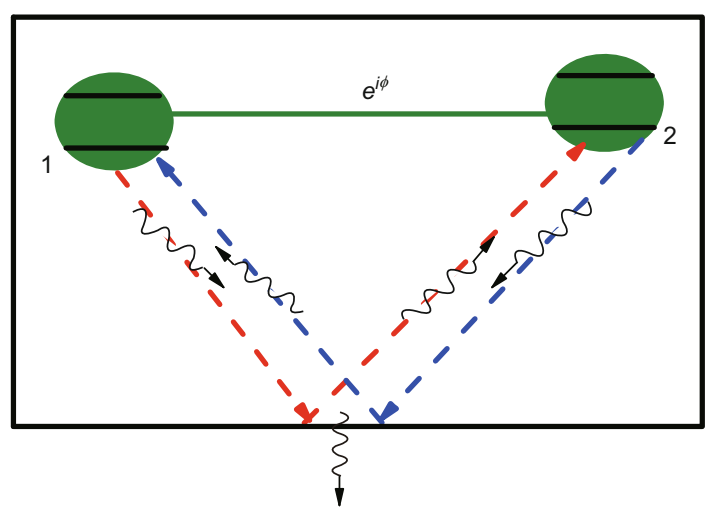

Figure 1 (Color online) Schematic diagram of two entangled two-level atoms coupled to a common environment. The excitation-emission by one atom will lead to the absorbtion of that excitation by another; therefore for each atom two opposite processes of excitation emission and absorbtion coexist at a time. A quantum interference can be induced by these two processes, while the relative phase $\phi$ in atomic entangled state determines the destructive or constructive feature of the interference.

\section{Model and solution}

Consider a pair of two-level atoms interacting with a common structured reservoir which can be modeled as the electromagnetic field inside a lossy cavity (see Figure 1). The Hamiltonian $H$ of the total system contains three parts $(\hbar=1)$ :

$$
\hat{H}=\hat{H}_{0}+\hat{H}_{\text {int }}+\hat{H}_{\text {dd }},
$$

where $H_{0}$ is the free Hamiltonian, $H_{\text {int }}$ denotes the atomreservoir coupling, and $H_{\mathrm{dd}}$ is responsible for the dipoledipole interaction:

$$
\begin{gathered}
\hat{H}_{0}=\omega_{1} \hat{\sigma}_{+}^{(1)} \hat{\sigma}_{-}^{(1)}+\omega_{2} \hat{\sigma}_{+}^{(2)} \hat{\sigma}_{-}^{(2)}+\sum_{k} \omega_{k} \hat{a}_{k}^{+} \hat{a}_{k} \\
\hat{H}_{\mathrm{int}}=\left(\alpha_{1} \hat{\sigma}_{+}^{(1)}+\alpha_{2} \hat{\sigma}_{+}^{(2)}\right) \sum_{k} g_{k} \hat{a}_{k}+H . c ., \\
\hat{H}_{\mathrm{dd}}=D\left(\hat{\sigma}_{+}^{(1)} \hat{\sigma}_{-}^{(2)}+\hat{\sigma}_{-}^{(1)} \hat{\sigma}_{+}^{(2)}\right) .
\end{gathered}
$$

Here, $\hat{\sigma}_{ \pm}^{j}$ and $\omega_{j}$ are the inversion operator and transition frequency of the atom $j=1,2, \hat{a}_{k}$ and $\hat{a}_{k}^{\dagger}$ are the annihilation and creation operators for the $k$ th mode of the reservoir with frequency $\omega_{k}$. As the coupling of an atom to the reservoir depends on the value of the cavity field at the atomic position, a dimensionless real constants $\alpha_{j}$ is introduced to individualize the atom: the actual coupling strength between the $j$ th atom and an $k$ th mode is thus measured by $\alpha_{j}\left|g_{k}\right|$. The static part of the dipole-dipole interaction is proportional to $D=\left[\vec{d} \cdot \vec{d}-3\left(\vec{d} \cdot \vec{r}_{12}\right)\left(\vec{d} \cdot \vec{r}_{12}\right) / r_{12}^{2}\right] r_{12}^{-3}$, where $\vec{r}_{12}=\vec{r}_{1}-\vec{r}_{2}$ is the relative position and $\vec{d}$ is the electric dipole moment of the atom. Though in calculation we include the dipole-dipole interaction of the two atoms, it is not a crucial factor for the appearance of quantum interference effect.

Assume that at time $t=0$ the cavity is empty (i.e. its state is $|\overline{\mathbf{0}}\rangle=\bigotimes_{k}\left|0_{k}\right\rangle_{r}$ with $\left|0_{k}\right\rangle_{r}$ the cavity state containing 0 excitations in mode $k$ ) and the two atoms are in the Bell-like state

$$
|\psi(0)\rangle=c_{1}(0)|e, g\rangle_{1,2}+c_{2}(0)|g, e\rangle_{1,2},
$$

with $\left|c_{1}(0)\right|^{2}+\left|c_{2}(0)\right|^{2}=1$. Therefore, the total system of atoms and reservoir contains only one excitation which may be initially in the first atom with a probability $\left|c_{1}(0)\right|^{2}$ or in the second atom with $\left|c_{2}(0)\right|^{2}$. Since $\left[\hat{H}, \sum_{k} \hat{a}_{k}^{+} \hat{a}_{k}+\hat{\sigma}_{+}^{(1)} \hat{\sigma}_{-}^{(1)}+\right.$ $\left.\hat{\sigma}_{+}^{(2)} \hat{\sigma}_{-}^{(2)}\right]=0$, the initial total state $|\Psi(0)\rangle=|\psi(0)\rangle \otimes|\overline{\mathbf{0}}\rangle$ evolves at time $t>0$ into

$$
\begin{aligned}
|\Psi(t)\rangle= & e^{-i \omega_{0} t}\left[c_{1}(t)|e, g\rangle_{1,2}+c_{2}(t)|g, e\rangle_{1,2}\right] \otimes|\overline{\mathbf{0}}\rangle \\
& +\sum_{k} c_{k}(t) e^{-i \omega_{k} t}\left|g, g, 1_{k}\right\rangle_{1,2, r}
\end{aligned}
$$

with $\left|1_{k}\right\rangle_{r}$ the reservoir state containing only one excitation in the $k$ th mode. Here, we have considered the two atoms have the same frequency, i.e. $\omega_{1}=\omega_{2}=\omega_{0}$. The equations of motion for the probability amplitudes take the form

$$
\dot{c}_{1}(t)=-i \alpha_{1} \sum_{k} g_{k} e^{-i\left(\omega_{k}-\omega_{0}\right) t} c_{k}(t)-i D c_{2}(t),
$$




$$
\begin{gathered}
\dot{c}_{2}(t)=-i \alpha_{2} \sum_{k} g_{k} e^{-i\left(\omega_{k}-\omega_{0}\right) t} c_{k}(t)-i D c_{1}(t), \\
\dot{c}_{k}(t)=-i g_{k}^{*} e^{i\left(\omega_{k}-\omega_{0}\right) t}\left[\alpha_{1} c_{1}(t)+\alpha_{2} c_{2}(t)\right] .
\end{gathered}
$$

Integrating eq. (9) with the initial condition $c_{k}(0)=0$ and inserting its solution into eqs. (7) and (8), one obtains the integro-differential equations for the amplitudes $c_{1}(t)$ and $c_{2}(t)$ :

$$
\begin{aligned}
\dot{c}_{1}(t)= & -\int_{0}^{t} \mathrm{~d} t^{\prime} \sum_{k}\left|g_{k}\right|^{2} e^{-i\left(\omega_{k}-\omega_{0}\right)\left(t-t^{\prime}\right)}\left[\alpha_{1}^{2} c_{1}\left(t^{\prime}\right)+\alpha_{1} \alpha_{2} c_{2}\left(t^{\prime}\right)\right] \\
& -i D c_{2}(t), \\
\dot{c}_{2}(t)= & -\int_{0}^{t} \mathrm{~d} t^{\prime} \sum_{k}\left|g_{k}\right|^{2} e^{-i\left(\omega_{k}-\omega_{0}\right)\left(t-t^{\prime}\right)}\left[\alpha_{1} \alpha_{2} c_{1}\left(t^{\prime}\right)+\alpha_{2}^{2} c_{2}\left(t^{\prime}\right)\right] \\
& -i D c_{1}(t) .
\end{aligned}
$$

The sum $\sum_{k}\left|g_{k}\right|^{2} e^{-i\left(\omega_{k}-\omega_{0}\right)\left(t-t^{\prime}\right)}$ in the above equations is recognized as nothing else but the reservoir correlation function $f\left(t-t^{\prime}\right)={ }_{r}\left\langle\overline{\mathbf{0}}\left|A(t) A^{\dagger}\left(t^{\prime}\right)\right| \overline{\mathbf{0}}\right\rangle_{r}$, with $A(t)=\sum_{j} g_{j} a_{j} e^{-i\left(\omega_{j}-\omega_{0}\right) t}$. In the limit of a large number of modes, the summation over the reservoir modes can be changed to an integration $\int \mathrm{d} \omega J(\omega) e^{-i\left(\omega-\omega_{0}\right)\left(t-t^{\prime}\right)}$, where $J(\omega)$ is referred to as the reservoir effective spectral density. In the continuum limit for the environment and by introducing the correlation function, eqs. (10) and (11) become

$$
\begin{aligned}
& \dot{c}_{1}(t)=-\int_{0}^{t} \mathrm{~d} t^{\prime} f\left(t-t^{\prime}\right)\left[\alpha_{1}^{2} c_{1}\left(t^{\prime}\right)+\alpha_{1} \alpha_{2} c_{2}\left(t^{\prime}\right)\right]-i D c_{2}(t), \\
& \dot{c}_{2}(t)=-\int_{0}^{t} \mathrm{~d} t^{\prime} f\left(t-t^{\prime}\right)\left[\alpha_{1} \alpha_{2} c_{1}\left(t^{\prime}\right)+\alpha_{2}^{2} c_{2}\left(t^{\prime}\right)\right]-i D c_{2}(t) .
\end{aligned}
$$

In the following we consider a realistic cavity whose photons can be leaked out through its nonperfect mirrors. In this case, the fundamental mode $\omega_{c}$ supported by the cavity displays a Lorentzian broadening due to the non-perfect reflectivity of the cavity mirrors. The effective spectral density of the intracavity field can be modeled as

$$
J(\omega)=\frac{W^{2}}{\pi} \frac{\lambda}{\left(\omega-\omega_{c}\right)^{2}+\lambda^{2}} .
$$

Laplace transforming both sides of eqs. (12) and (13), with $J(\omega)$ given by eq. (14), yields

$$
\begin{aligned}
& \widetilde{c}_{1}(s)=\frac{\left[s(s+\lambda)+R^{2} r_{2}^{2}\right] c_{1}(0)-\left[R^{2} r_{1} r_{2}+i D(s+\lambda)\right] c_{2}(0)}{s^{2}(s+\lambda)+R^{2} s+D^{2}(s+\lambda)-2 i R^{2} r_{1} r_{2} D}, \\
& \widetilde{c}_{2}(s)=\frac{\left[s(s+\lambda)+R^{2} r_{1}^{2}\right] c_{2}(0)-\left[R^{2} r_{1} r_{2}+i D(s+\lambda)\right] c_{1}(0)}{s^{2}(s+\lambda)+R^{2} s+D^{2}(s+\lambda)-2 i R^{2} r_{1} r_{2} D},
\end{aligned}
$$

where the notation $\widetilde{f}(s)=\mathcal{L}[f(t)]=\int_{0}^{t} f(t) e^{-s t} \mathrm{~d} t$ and the following dimensionless quantities have been introduced for convenience: the collective coupling constant $\alpha_{T}=$ $\sqrt{\alpha_{1}^{2}+\alpha_{2}^{2}}$, the relative coupling strengths $r_{1(2)}=\alpha_{1(2)} / \alpha_{T}$, and the vacuum Rabi frequency $R=W \alpha_{T}$. Not that $r_{1}^{2}+r_{2}^{2}=$
1, by definition. Applying the inverse Laplace transforms on eqs. (15) and (16), we get the time-dependent solutions:

$$
c_{j}(t)=\sum_{n} \lim _{s \rightarrow s_{n}}\left(s-s_{n}\right) \widetilde{c}_{j}(s) e^{s_{n} t},
$$

with $j=1,2$ and $s_{n}$ a pole of $\widetilde{c}_{j}(s)$. In the absence of dipoledipole interaction with $D=0$, the analytic expressions of $c_{1}(t)$ and $c_{2}(t)$ can be obtained as

$$
\begin{aligned}
& c_{1}(t)=\left[r_{2}^{2}+r_{1}^{2} \mathcal{E}(t)\right] c_{1}(0)+r_{1} r_{2}[\mathcal{E}(t)-1] c_{2}(0), \\
& c_{2}(t)=\left[r_{1}^{2}+r_{2}^{2} \mathcal{E}(t)\right] c_{2}(0)+r_{1} r_{2}[\mathcal{E}(t)-1] c_{1}(0),
\end{aligned}
$$

in which $\mathcal{E}(t)=e^{-\lambda t / 2}\left[\cosh \left(\frac{\Omega t}{2}\right)+\frac{\lambda}{\Omega} \sinh \left(\frac{\Omega t}{2}\right)\right]$ and $\Omega=$ $\sqrt{\lambda^{2}-4 R^{2}}$. In the $\{|e, e\rangle,|e, g\rangle,|g, e\rangle,|g, g\rangle\}$ basis, the reduced density operator for the two atoms is given by

$$
\rho_{12}=\left(\begin{array}{cccc}
0 & 0 & 0 & 0 \\
0 & \left|c_{1}(t)\right|^{2} & c_{1}(t) c_{2}^{*}(t) & 0 \\
0 & c_{1}^{*}(t) c_{2}(t) & \left|c_{2}(t)\right|^{2} & 0 \\
0 & 0 & 0 & 1-\left|c_{1}(t)\right|^{2}-\left|c_{1}(t)\right|^{2}
\end{array}\right) .
$$

We adopt the concurrence [42] $C(t)$ as the measure of atomic entanglement, which takes the form

$$
C(t)=2\left|c_{1}(t) c_{2}^{*}(t)\right|
$$

In this work, we are concerned with the quantum interference induced by two entangled atoms. The feature of the interference is closely related to the relative phase of atomic initial entangled state (5); therefore we reexpress $c_{1}(0)$ and $c_{2}(0)$ as

$$
c_{1}(0)=\sin \theta, \quad c_{2}(0)=\cos \theta e^{i \phi},
$$

with $\theta \in[0, \pi / 2]$ and $\phi \in[0, \pi]$. Though in essence the initial state of the atoms is characterized by both the parameters $\theta$ and $\phi$, the initial excited-state population of the atoms as well as its initial concurrence $C(0)=2\left|c_{1}(0) c_{2}^{*}(0)\right|=2 \sin \theta \cos \theta$ are determined only by $\theta$. In this connection, one may ask for the definite value of $\theta$, how the the relative phase $\phi$ influences the subsequent dynamics of the atoms? We shall show that a quantum interference effect can be induced due to the interaction of the entangled atoms with the common environment. The destructive and constructive features of the interference are determined by the relative phase in the initial entangled state of the atoms. The excited-state population trapped in the atoms and atomic stationary entanglement are hence influenced strongly by the quantum interference.

\section{Quantum interference}

At first, we consider the time evolution of excited-state population of one atom, say, the first atom, which for $D=0$ can be expressed by virtue of eq. (18) as

$$
\left|c_{1}(t)\right|^{2}=T_{1}(t)+T_{2}(t)+T_{3}(t)
$$


with

$$
\begin{aligned}
& T_{1}(t)=\left[r_{2}^{2}+r_{1}^{2} \mathcal{E}(t)\right]^{2}\left|c_{1}(0)\right|^{2}, \\
& T_{2}(t)=r_{1}^{2} r_{2}^{2}[\mathcal{E}(t)-1]^{2}\left|c_{2}(0)\right|^{2}, \\
& T_{3}(t)=2 r_{1} r_{2}\left[r_{2}^{2}+r_{1}^{2} \mathcal{E}(t)\right][\mathcal{E}(t)-1] \sin \theta \cos \theta \cos \phi,
\end{aligned}
$$

where we have employed the expression (22) for the term $T_{3}(t)$. Obviously, the excited-state population of an individual atom is composed of three parties, i.e. $T_{1}(t), T_{2}(t)$ and $T_{3}(t)$. The term $T_{1}(t)(24)$ represents the emission of excitation that lies initially in the first atom with a probability $\left|c_{1}(0)\right|^{2}$. On the contrary, $T_{2}(t)$ denotes the absorbtion of excitation that lies initially in the second atom by the first atom. Actually, the single excitation may initially be in the second atom with a probability $\left|c_{2}(0)\right|^{2}$, which will be emitted to the cavity and then be absorbed partially by the first atom due to the interaction with the cavity. That is, the excitationemission of one atom leads to the excitation-absorbtion of another in the presence of a common environment. As a result, two opposite processes of excitation emission and absorbtion coexist for the first atom, by which a quantum interference is induced and represented by the term $T_{3}(t)$. From eq. (26), one can see the interference term $T_{3}(t)$ is dependent on the value of $\phi$, i.e. the relative phase in atomic initial entangled state, which determines the features of the quantum interference: for $0 \leqslant \phi<\pi / 2$, we have $T_{3}(t)<0$ corresponding to the destructive interferences, while for $\pi / 2<\phi \leqslant \pi$, we instead have $T_{3}(t)>0$ corresponding to the constructive interferences. For $\phi=\pi / 2$, we are left with $T_{3}(t)=0$, which means the two processes of excitation emission and absorbtion are independent so that the quantum interference does not occur. Though we focus on the first atom here, the preceding discussion is suitable for the second atom as well.

The quantum inferences under various values of the relative phase $\phi$ are bound to influence the dynamics of atomic excited-state population during the time-evolution. Here, we are more interested in the excited-state population trapped in the atomic system in the long-time limit, which for $t \rightarrow \infty$ reads

$$
\begin{aligned}
\left|c_{1}^{s}\right|^{2} & \equiv \lim _{t \rightarrow \infty}\left|c_{1}(t)\right|^{2} \\
& =r_{2}^{4} \sin ^{2} \theta+r_{1}^{2} r_{2}^{2} \cos ^{2} \theta-2 r_{1} r_{2}^{3} \sin \theta \cos \theta \cos \phi .
\end{aligned}
$$

As shown by eq. (27), for any fixed initial entanglement in terms of $\theta$ and relative coupling strengthes $r_{1}$ and $r_{2}$, the asymptotical excited-state population $\left|c_{1}^{s}\right|^{2}$ of the first atom is proportional to $\phi \in[0, \pi]$. This result applies also to the second atom; hence the total excited-state population of the two atoms can be trapped to a greater extent for a bigger $\phi$.

In Figure 2, we plot the time evolution of $\left|c_{1}(t)\right|^{2}$ under various values of $\phi$ in both Markovian and non-Markovian reservoirs. In both regimes, the asymptotical values of $\left|c_{1}(t)\right|^{2}$, i.e. $\left|c_{1}^{s}\right|^{2}$, increase with an increase of $\phi$. Compared to the case of no quantum interference $(\phi=\pi / 2)$, the steady value of $\left|c_{1}(t)\right|^{2}$ is reduced by the destructive quantum interference (e.g. $\phi=0, \pi / 4$ ), while enhanced by the constructive quantum interference (e.g. $\phi=3 \pi / 4, \pi$ ). The excited-state population can exceed its initial value for a biggish $\phi$, such as $\phi=\pi / 2,3 \pi / 4, \pi$ here. The net increase of the excited-state population, i.e. $\left|c_{1}^{s}\right|^{2}-\left|c_{1}(0)\right|^{2}$, is obviously transferred from the cavity, to which the second atom emits. It can be verified the asymptotical excited-state population $\left|c_{2}^{s}\right|^{2}$ of the second atom also increases with an increase of $\phi$, although it cannot exceed its initial value $\left|c_{2}(0)\right|^{2}$ for the present choice of parameters. The inset in Figure 2(a) shows the dynamics of $T_{1}(t), T_{2}(t)$ and $T_{3}(t)$, the three constituents of $\left|c_{1}(t)\right|^{2}$. The terms $T_{1}(t)$ and $T_{2}(t)$ are independent of $\phi$, while the interference term $T_{3}(t)$ is closely dependent on $\phi$, which varies from a negative value for $\phi=0(\phi<\pi / 2)$ to null for $\phi=\pi / 2$ and then to a positive value for $\phi=\pi(\phi>\pi / 2)$. In the inset of Figure 2(b), we have shown the dynamics of $\left|c_{1}(t)\right|^{2}$ in the initial period for the non-Markovian regime. Though the asymptotical values of $\left|c_{1}(t)\right|^{2}$ are proportional to $\phi$, it is

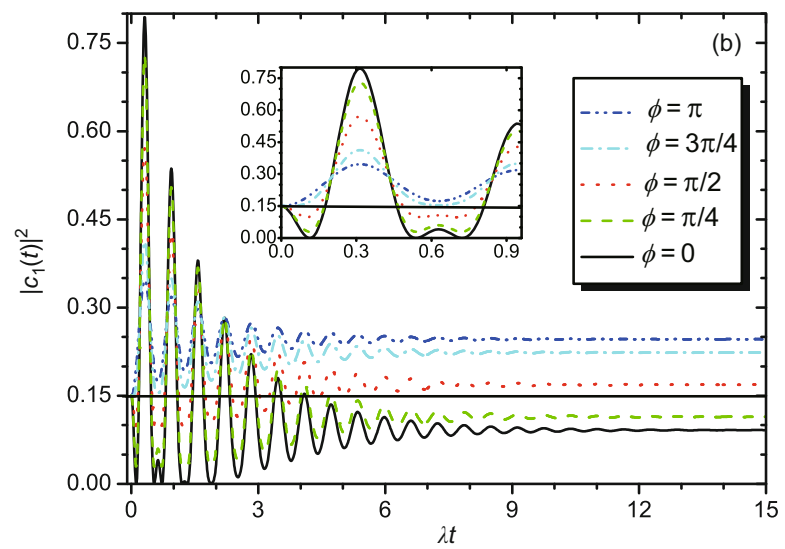

Figure 2 (Color online) Time evolution of excited-state population $\left|c_{1}(t)\right|^{2}=T_{1}(t)+T_{2}(t)+T_{3}(t)$ of the first atom for $\sin \theta=\sqrt{\frac{3}{20}}, \cos \theta=\sqrt{\frac{17}{20}}$ and different relative phases $\phi$ in (a) the Markovian $(R / \lambda=0.1)$ and (b) non-Markovian $(R / \lambda=10)$ reservoirs. The other parameters are $D=0$ and $r_{1}=\sqrt{3} / 2$, $r_{2}=1 / 2$. The inset in (a) shows the dynamics of $T_{1}(t), T_{2}(t)$ (black dashed lines) and that of $T_{3}(t)$ for $\phi=0, \pi / 2, \pi$ (orange solid lines). The inset in (b) shows the dynamics of $\left|c_{1}(t)\right|^{2}$ in the initial period. 
not the case in the short time: due to the oscillation of $\left|c_{1}(t)\right|^{2}$, the values of which are alternate for the big and the small $\phi$.

\section{Stationary entanglement}

The expression (21) of atomic concurrence shows that only when neither of $\left|c_{1}(t)\right|$ and $\left|c_{2}(t)\right|$ approaches zero in the time limit can a nonzero stationary value of $C(t)$ exists. It can be verified that when $D=0$, i.e. in the absence of dipoledipole interaction, there always exists a stationary entanglement $C_{s}=C(t \rightarrow \infty)$, which can be obtained by virtues of eqs. (18), (19) and (22) as

$$
C_{s}=2 r_{1} r_{2}\left(r_{2}^{2} \sin ^{2} \theta+r_{1}^{2} \cos ^{2} \theta-2 r_{1} r_{2} \sin \theta \cos \theta \cos \phi\right) .
$$

Nevertheless, if $D \neq 0$, the condition for the existence of a stationary entanglement is that the two atoms should have equivalent coupling strengths to the cavity mode [26] and the stationary entanglement is given in (28) with $r_{1}=r_{2}$. Though the dipole-dipole interaction influences the detailed process of entanglement evolution, it does not change the stationary entanglement once the parameters $\theta, \phi$ and $r_{1}=r_{2}$ are given. Therefore, we shall concentrate on the general situation of $D=0$ in the following consideration.

(a)


Our first observation from eq. (28) is that besides the relative coupling strengthes of the two atoms to the cavity mode, the stationary entanglement will be determined by the initial state of the system in terms of both $\theta$ and $\phi$. For any definite values of $r_{1}, r_{2}$ and $\theta \neq 0, \pi / 2$, the maximal (minimal) stationary entanglement always correspond to an initial relative phase of $\phi=\pi(\phi=0)$. As an example, for three particular cases of $\phi=0, \pi / 2, \pi$, the stationary entanglement takes the forms $C_{s}^{0}=2 r_{1} r_{2}\left(r_{2}^{2} \sin ^{2} \theta+\right.$ $\left.r_{1}^{2} \cos ^{2} \theta-2 r_{1} r_{2} \sin \theta \cos \theta\right), C_{s}^{\pi / 2}=2 r_{1} r_{2}\left(r_{2}^{2} \sin ^{2} \theta+r_{1}^{2} \cos ^{2} \theta\right)$ and $C_{s}^{\pi}=2 r_{1} r_{2}\left(r_{2}^{2} \sin ^{2} \theta+r_{1}^{2} \cos ^{2} \theta+2 r_{1} r_{2} \sin \theta \cos \theta\right)$ and thus the inequality $C_{s}^{0}<C_{s}^{\pi / 2}<C_{s}^{\pi}$ holds for any initial nonzero entanglement. The result coincides with the relation between the total excited-state population trapped in the two atoms and the relative phase. Therefore, an explicit link is constructed between the stationary entanglement and excitedstate population of the two atoms. That is, it is due to the relative phase dependent quantum interference that lead to the possible increase of both stationary excited-state population and entanglement. In Figure 3, we plot the dynamics of entanglement and total excited-state population of the two atoms with respect to a fixed initial concurrence and different relative phases in both Markovian and non-Markovian reservoirs.

(b)
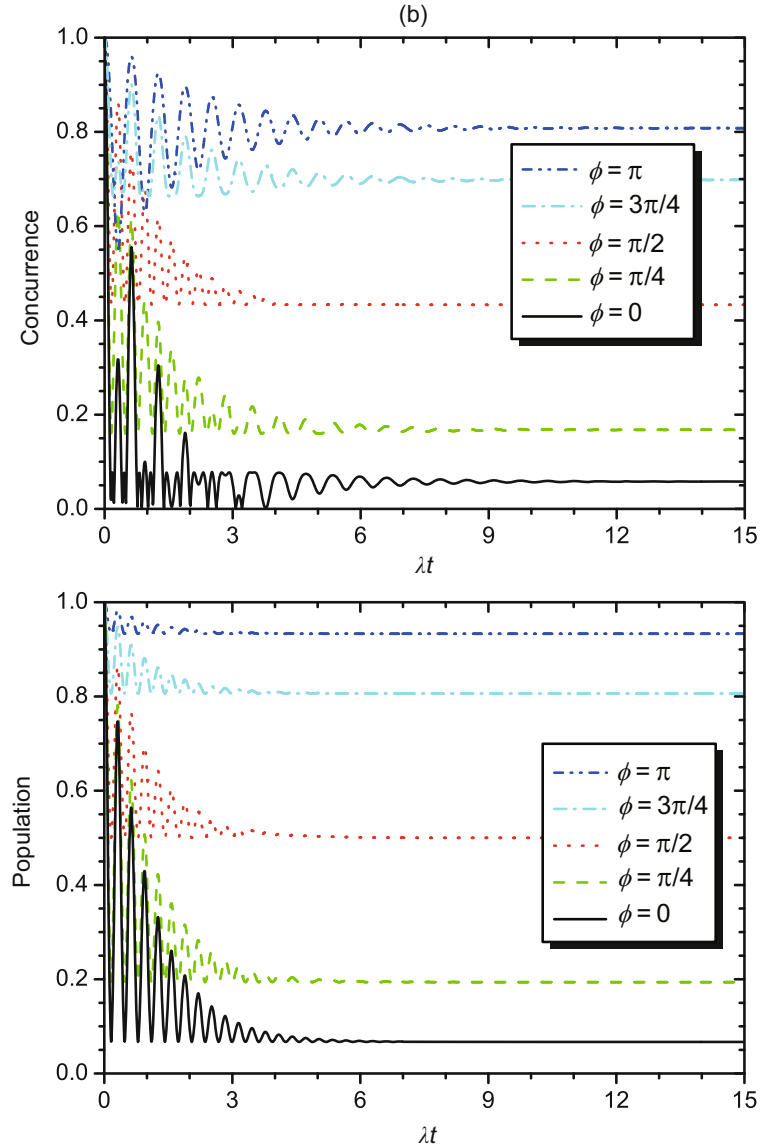

Figure 3 (Color online). Time evolution of the concurrence and excited-state population of the two atoms for the initial entangled state with a fixed $\theta=\pi / 4$ and different relative phases $\phi$ in (a) the Markovian $(R / \lambda=0.1)$ and (b) non-Markovian $(R / \lambda=10)$ reservoirs. We have considered the case of no dipole-dipole interaction $D=0$ and $r_{1}=\sqrt{3} / 2, r_{2}=1 / 2$. 
The figure clearly show the increases of both stationary entanglement and excited-state population with an increase of $\phi$ from zero to $\pi$. One can also note that though both entanglement and excited-state population exhibit distinct dynamics behaviors in Markovian and non-Markovian reservoirs, their stationary values are the same in the two regimes. This can be verified from the formulae (27) and (28) that stationary excited-state population and entanglement are determined only by the relative coupling strengthes $r_{1}, r_{2}$ and the initial state in terms of both $\theta$ and $\phi$, while independent to concrete parameters of the reservoir.

Our second observation is that for definite values of $r_{1}, r_{2}$ and $\theta$ if they satisfy the condition

$$
-1 \leqslant \frac{1}{2}\left(\frac{r_{1} \cos \theta}{r_{2} \sin \theta}+\frac{r_{2} \sin \theta}{r_{1} \cos \theta}-\frac{1}{r_{1}^{2} r_{2}^{2}}\right) \leqslant 1,
$$

then the stationary entanglement of the two atoms can equal and even exceed their initial entanglement when the relative phase $\phi$ further satisfies the relation

$$
\cos \phi \leqslant \frac{1}{2}\left(\frac{r_{1} \cos \theta}{r_{2} \sin \theta}+\frac{r_{2} \sin \theta}{r_{1} \cos \theta}-\frac{1}{r_{1}^{2} r_{2}^{2}}\right),
$$

where the equality and inequality denote respectively the stationary entanglement be equal to and larger than the initial entanglement. The fact that the stationary entanglement can asymptotically reach the initial entanglement does not necessarily mean the entangled state is the decoherence-free eigenstate of the Hamiltonian of the composite system. Actually, the eigenstate is a particular case of (29) and (30) corresponding to $\cos \phi=\frac{1}{2}\left(\frac{r_{1} \cos \theta}{r_{2} \sin \theta}+\frac{r_{2} \sin \theta}{r_{1} \cos \theta}-\frac{1}{r_{1}^{2} r_{2}^{2}}\right)=-1$, i.e. $r_{1}=\cos \theta$, $r_{2}=\sin \theta$ and $\phi=\pi$. Namely, the decoherence-free state has the form $\left|\psi_{-}\right\rangle=r_{2}|e, g\rangle-r_{1}|g, e\rangle$. In Figure 4, we show the growth of stationary entanglement of the two atoms over their initial entanglement by adjusting the relative phase of the initial entangled state. Here, we choose $\sin \theta=\sqrt{\frac{1}{10}}$, $\cos \theta=\sqrt{\frac{9}{10}}$ (i.e. $\left.C(0)=2 \sin \theta \cos \theta=0.6\right)$ and $r_{1}=\sqrt{3} / 2$,

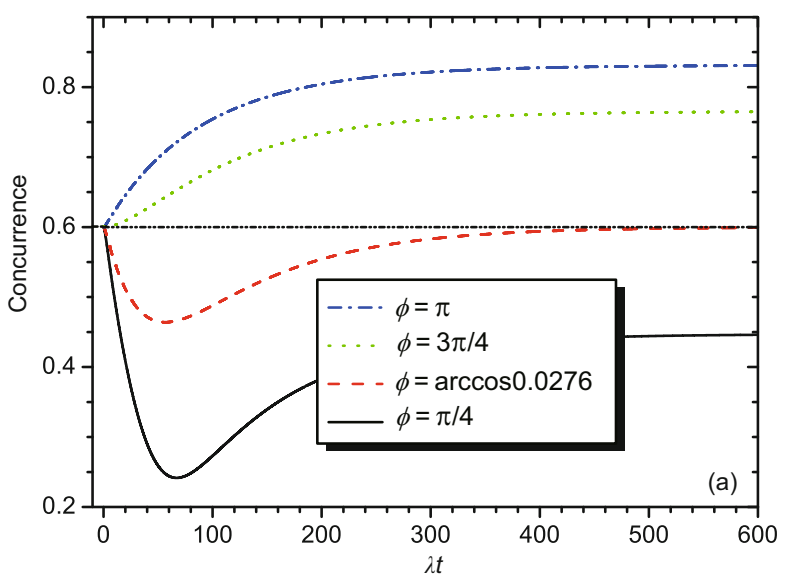

$r_{2}=1 / 2$, under which the condition in eq. (29) is satisfied and the right hand side of eq. (30) is approximatively equal to 0.0276. According to the restriction of eq.(30) and as shown in Figure 4 , when $\cos \phi \leqslant 0.0276$ (i.e. $\phi \geqslant \arccos 0.0276$ ) the stationary entanglement can reach and exceed the initial value $C(0)=0.6$. In fact, the existence of a stationary entangled state is due to the decoherence-free space of the model. Therefore, given certain initial states of the system their stationary entanglement can be enhanced. Here, from another perspective, we present a possible explanation for the growth of atoms' stationary entanglement over the initial value. From eq. (21), atoms' entanglement in the evolution is determined by the product of $\left|c_{1}(t)\right|$ and $\left|c_{2}(t)\right|$, i.e. the excited state populations of the two atoms. Under the conditions (29) and (30) we have proposed, the single excitation can be redistributed in the two atoms in such a way that the values of $\left|c_{1}(t)\right|$ and $\left|c_{2}(t)\right|$ are more even for $t \rightarrow \infty$ and thus the stationary entanglement is correspondingly improved. Taking the parameters used in Figure 4 as an example, at the beginning, $\left|c_{0}\right|=|\sin \theta|=\sqrt{\frac{1}{10}} \approx 0.32$ and $\left|c_{2}(0)\right|=\left|\cos \theta e^{i \phi}\right|=\sqrt{\frac{9}{10}} \approx 0.95$ (i.e. the initial concurrence $C(0)=0.6)$. In the long-time limit (e.g. $\lambda t=15$ for the strong coupling regime), we get that $\left|c_{1}(t)\right| \approx 0.49$ and $\left|c_{2}(t)\right| \approx 0.85$ for $\phi=\pi$ (corresponding to the blue line in Figure 4(b)), which are more even in comparison to the initial time and thus lead to an increased stationary entanglement $C(t) \approx 0.83$ over the initial value.

\section{Conclusion}

In conclusion, we have studied a novel quantum interference induced by two entangled atoms coupled to a common environment. The relative phase in the initial entangled state determines the constructive and destructive features of the interference. It is shown the trapped excited-state population of

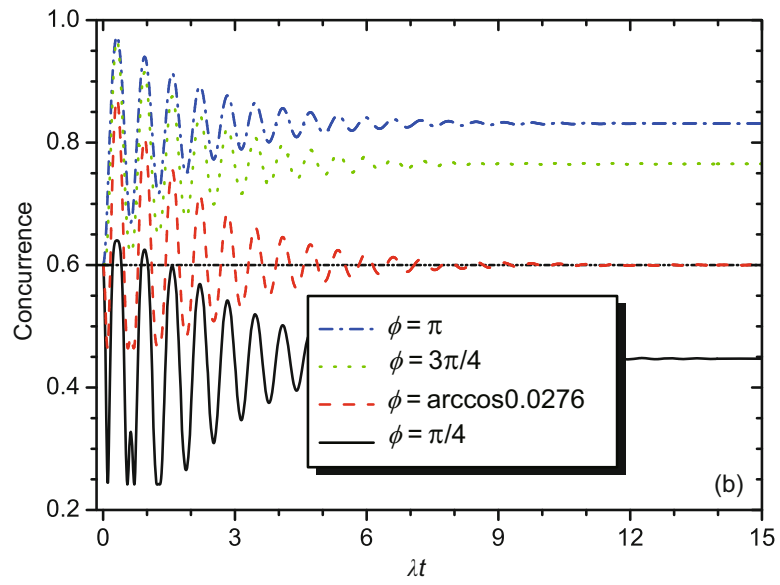

Figure 4 (Color online) Time evolution of atomic entanglement for a fixed initial concurrence $C(0)=0.6$, i.e. $\sin \theta=\sqrt{\frac{1}{10}}$, $\cos \theta=\sqrt{\frac{9}{10}}$ and different relative phases $\phi$ in (a) the Markovian $(R / \lambda=0.1)$ and (b) non-Markovian $(R / \lambda=10)$ reservoirs. The case of no dipole-dipole interaction $D=0$ and $r_{1}=\sqrt{3} / 2, r_{2}=1 / 2$ is considered. 
both the individual atom and the total atoms are closely related to the features of quantum interference. An explicit link between the trapped excited-state population and the stationary entanglement is constructed. It is shown atomic stationary entanglement can be promoted by adjusting the values of the relative phases. The stationary entanglement of the atoms can reach and exceed their initial value under a given condition.

This work was supported by the National Natural Science Foundation of China (11204156, 10947006 and 61178012), the Specialized Research Fund for the Doctoral Program of Higher Education (20093705110001) and the Scientific Research Foundation of Qufu Normal University for Doctors (BDQD20100203).

1 Nielsen M I, Chuang I L. Quantum Computation and Quantum Information. Cambridge: Cambridge University Press, 2000

2 Salemian S, Mohammadnejad S. An error-free protocol for quantum entanglement distribution in long-distance quantum communication. Chin Sci Bull, 2011, 56: 618-625

3 Wang Y Z, Hou J C, Guo Y. An entanglement criterion for states in infinite-dimensional multipartite quantum systems. Chin Sci Bull, 2012, 57: 1643-1647

4 Ding S C, Jin Z. Review on the study of entanglement in quantum computation speedup. Chin Sci Bull, 2007, 52: 2161-2166

5 Breuer H P, Petruccione F. The Theory of Open Quantum Systems. Oxford: Oxford University Press, 2002

6 Yu T, Eberly J H. Finite-time disentanglement via spontaneous emission. Phys Rev Lett, 2004, 93: 140404

7 Eberly J H, Yu T. The end of an entanglement. Science, 2007, 316: 555-557

8 Yu T, Eberly J H. Sudden death of entanglement. Science, 2009, 323: 598-601

9 Almeida M P, de Melo F, Hor-Meyll M, et al. Environment-induced sudden death of entanglement. Science, 2007, 316: 579-582

10 Jiménez F O, Valdes-Hernandez A, Lguilar G, et al. Experimental investigation of dynamical invariants in bipartite entanglement. Phys Rev A, 2012, 85: 012314

11 Viviescas C, Guevara I, Carvalho A R R, et al. Entanglement dynamics in open two-qubit systems via diffusive quantum trajectories. Phys Rev Lett, 2010, 105: 210502

12 Chen Q H, Yang Y, Liu T, et al. Entanglement dynamics of two independent Jaynes-Cummings atoms without the rotating-wave approximation. Phys Rev A, 2010, 82: 052306

13 Xu J S, Li C F, Xu X Y, et al. Experimental characterization of entanglement dynamics in noisy channels. Phys Rev Lett, 2009, 103: 240502

14 Salles A, de Melo F, Almeida M P, et al. Experimental investigation of the dynamics of entanglement: Sudden death, complementarity, and continuous monitoring of the environment. Phys Rev A, 2008, 78: 022322

15 Bellomo B, Franco R L, Compagno G. Entanglement dynamics of two independent qubits in environments with and without memory. Phys Rev A, 2008, 77: 032342

16 Bellomo B, Franco R L, Compagno G. Non-Markovian effects on the dynamics of entanglement. Phys Rev Lett, 2007, 99: 160502

17 Man Z X, Xia Y J, An N B. Entanglement measure and dynamics of multiqubit systems: Non-Markovian versus Markovian and generalized monogamy relations. New J Phys, 2010, 12: 033020
18 Dodd P J, Halliwell J. Disentanglement and decoherence by open system dynamics. Phys Rev A, 2004, 69: 052105

19 Santos M F, Milman P, Davidovivh L, et al. Direct measurement of finite-time disentanglement induced by a reservoir. Phys Rev A, 2006, 73: 040305(R)

20 Sainz I, Bjök G. Entanglement invariant for the double JaynesCummings model. Phys Rev A, 2007, 76: 042313

21 Ficek Z, Tanas R. Dark periods and revivals of entanglement in a twoqubit system. Phys Rev A, 2006, 74: 024304

22 Zyczkowski K, Horodecki P, Horodecki M, et al. Dynamics of quantum entanglement. Phys Rev A, 2001, 65: 012101

23 Paz J P, Roncaglia A J. Dynamics of the entanglement between two oscillators in the same environment. Phys Rev Lett, 2008, 100: 220401

24 Mazzola L, Maniscalco S, Piilo J, et al. Sudden death and sudden birth of entanglement in common structured reservoirs. Phys Rev A, 2009, 79: 042302

25 Qian Y, Zhang Y Q, Xu J B. Amplifying stationary quantum discord and entanglement between a superconducting qubit and a data bus by timedependent electromagnetic field. Chin Sci Bull, 2012, 57: 1637-1642

$26 \mathrm{Li} \mathrm{Y,} \mathrm{Zhou} \mathrm{J,} \mathrm{Guo} \mathrm{H.} \mathrm{Effect} \mathrm{of} \mathrm{the} \mathrm{dipole-dipole} \mathrm{interaction} \mathrm{for} \mathrm{two}$ atoms with different couplings in a non-Markovian environment. Phys Rev A, 2009, 79: 012309

27 An N B, Kim J, Kim K. Entanglement dynamics of three interacting two-level atoms within a common structured environment. Phys Rev A, 2011, 84: 022329

28 Han F. Entanglement dynamics and transfer in a double JaynesCummings model. Chin Sci Bull, 2010, 55: 1758-1762

29 Yue H D, Zhang Y, Gong J. Quantum discord in three-spin XXZ chain with three-spin interaction. Sci China Phys Mech Astron, 2012, 55: 1641-1645

30 Yu X Y, Li J H, Li X B. Atom-atom entanglement characteristics in fiber-connected cavities system within the double-excitation space. Sci China Phys Mech Astron, 2012, 55: 1813-1819

31 Ai Q, Wang Y D, Long G L, et al. Two mode photon bunching effect as witness of quantum criticality in circuit. Sci China Ser G-Phys Mech Astron, 2009, 52: 1898-1905

32 Ye M Y, Lin X M, Bai Y K, et al. Entanglement charge of thermal states. Sci China Phys Mech Astron, 2012, 55: 666-670

33 Ma X S, Ren M F, Zhao G X, et al. Effect of decoherence from a spin environment on the entanglement dynamics of two-qutrit states. Sci China Phys Mech Astron, 2011, 54: 1833-1838

34 Guo K T, Liang M C, Xu H Y, et al. Entanglement in a two-spin (1/2, $3 / 2$ ) mixed-spin Heisenberg XX chain with an inhomogeneous external magnetic field. Sci China Phys Mech Astron, 2011, 54: 491-495

35 Maniscalco S, Francica F, Zaffino R L, et al. Protecting entanglement via the quantum Zeno effect. Phys Rev Lett, 2008, 100: 090503

36 Bellomo B, Franco R L, Maniscalco S, et al. Entanglement trapping in structured environments. Phys Rev A, 2008, 78: 060302(R)

37 Das S, Agarwal G S. Protecting bipartite entanglement by quantum interferences. Phys Rev A, 2010, 81: 052341

38 Sete E A, Das S. Quantum interference in timed Dicke basis and its effect on bipartite entanglement. Phys Rev A, 2011, 83: 042301

39 An N B, Kim J, Kim K. Nonperturbative analysis of entanglement dynamics and control for three qubits in a common lossy cavity. Phys Rev A, 2010, 82: 032316

40 Li M, Fei S M, Li-Jost X Q. Bell inequality, separability and entanglement distillation. Chin Sci Bull, 2011, 56: 945-954

41 Agarwal G S. Quantum Statistical Theories of Spontaneous Emission and their Relation to Other Approaches. Berlin: Springer, 1974

42 Wootters W K. Entanglement of formation of an arbitrary state of two qubits. Phys Rev Lett, 1998, 80: 2245-2248

Open Access This article is distributed under the terms of the Creative Commons Attribution License which permits any use, distribution, and reproduction in any medium, provided the original author(s) and source are credited. 